\title{
Prise en charge en réseau des patients insuffisants cardiaques
}

\author{
P. Mohacsi*
}

\section{Résumé}

L'insuffisance cardiaque est une entité nosologique qui requiert, pour une prise en charge efficace, la collaboration concertée de différents «acteurs» du système de santé. Nous proposons une prise en charge en réseau, englobant le médecin traitant - qui joue le rôle de plaque tournante -, les spécialistes, le personnel infirmier et l'entourage du patient.

Patients are patients, not customers Patients need care, not management [1]

\section{Introduction}

L'insuffisance cardiaque est un problème croissant en matière de santé publique. Au cours des dernières années, on constate une augmentation de la prévalence et de l'incidence de l'insuffisance cardiaque [2]. Cette évolution s'explique par les améliorations survenues dans le diagnostic, ainsi que par la prolongation de l'espérance de vie des patients cardio-vasculaires, grâce aux meilleures possibilités de traitement [3]. On observe en outre un vieillissement progressif de l'ensemble de la population [4]. A l'heure actuelle, environ $15 \%$ de la population est âgée de plus de 65 ans. Pour l'horizon 2040, on s'attend en Suisse à une structure démographique comportant environ $25 \%$ de personnes âgées de plus de 65 ans $[4,5]$. Outre la charge financière qu'elle représente pour nos institutions sociales (AVS, caisses de pension), cette évolution imposera très vraisemblablement aussi un nouveau choix de priorités en politique de la santé.

A l'heure actuelle, les affections cardio-vasculaires demeurent la principale cause de décès de la population suisse [6]. Dès l'âge de 65 ans, l'insuffisance cardiaque est la cause la plus fréquente d'hospitalisation [7].

Actuellement, on estime à environ 150000 le nombre de patients ${ }^{* *}$ qui souffrent d'insuffisance cardiaque, et à 10000 le nombre de nouveaux cas par année [8]. 80-90\% de ces patients présentent une insuffisance cardiaque légère à modérée. Ils sont pour la plupart pris en charge par les méde- cins de famille, et non par des centres spécialisés. C'est donc au médecin de premier recours qu'incombe l'autorité thérapeutique. 6-7\% environ des insuffisants cardiaques sont en classe fonctionnelle NYHA IV. Ces patients sévèrement atteints présentent d'une part une détérioration de leur qualité de vie, mais d'autre part ce sont eux aussi qui engendrent la majeure partie des coûts, en raison de leurs fréquentes hospitalisations. A l'avenir, on peut espérer détecter les sujets ayant un profil de risque cardio-vasculaire élevé avant même de déceler une affection cardiaque organique, symptomatique ou non (stade A de la nouvelle classification de l'American Heart Association [9]). Le «traitement» de personnes asymptomatiques, qui ne présentent rien de plus qu'un profil à risque, devrait de préférence consister en mesures thérapeutiques non-médicamenteuses. Le traitement médicamenteux n'est pas dénué de problèmes, car il peut tout d'abord entraîner des effets secondaires et ensuite parce qu'il génère des frais considérables (voir l'exemple des statines). Enfin, le «citoyen suisse moyen» n'est aujourd'hui pas très enclin à surveiller sa tension artérielle, sa glycémie, ses lipides et son poids ou à s'abstenir de tabac, en premier lieu peut-être parce que ses primes d'assurance-maladie ne sont pas pondérées en fonction des facteurs de risque évitables. A vrai dire, en l'absence d'un contrôle adéquat, l'introduction d'un tel système de primes pondérées reste difficile à réaliser.

Le problème croissant de l'insuffisance cardiaque en termes de santé publique et la prise en charge globale de chaque patient particulier requièrent non seulement la collaboration des médecins traitants, des spécialistes et de l'hôpital, mais aussi celle des autres «prestataires de soins», ainsi que du patient lui-même et de son entourage. Cela conduit logiquement à la mise en place d'un concept de prise en charge en réseau. Dans cet article, nous aimerions présenter les arguments en faveur d'un réseau et soumettre à consultation un concept que nous espérons attrayant.

Il n'est pas rare que les réseaux suscitent chez le médecin traitant la crainte que soit remise en question la prise en charge du patient comme personne globale. 


\section{«Managed Care» et «network» à I'américaine}

«Managed Care» et «network» résonnent comme des slogans. Ils font partie de la cosmologie moderne de notre vie imprégnée de «dot.com» et de «SMS», tout comme «new private or public management», «controlling» ou «certification ISO». Evitons la question de savoir si cette évolution, généralement en provenance du monde anglosaxon, est bonne. Toujours est-il que de nombreuses valeurs encore importantes il y a 20 ans pour les Suisses ont ainsi sombré dans l'oubli.

Le «Managed Care», en particulier sous la forme de HMOs (Health Maintenance Organizations), trouve son origine dans des modèles qui ont été introduits en Californie en 1930 en réponse à la forte dépression économique de l'époque [10]. Mais ce n'est que dans les années 80 et 90 que le «managed care» a connu son véritable boom. Il était censé représenter le principal instrument destiné à lutter contre l'augmentation frénétique des coûts de la santé aux Etats-Unis [11]. En Suisse, des programmes de soins coordonnés existent notamment pour les affections respiratoires (BPCO [12], asthme), pour le diabète, ainsi qu'en oncologie et en rhumatologie.

Depuis quelques années, les voix se multiplient aux Etats-Unis pour prophétiser la fin du «managed care» [13, 14]. Récemment, Paul Ellwood, un médecin du Wyoming, constatait que «la politique américaine de la santé n'est pas faite de grandes réformes, mais d'une expérimentation sociale perpétuelle, pour ainsi dire un procédé d'apprentissage par l'erreur». Les HMOs, ancrées dans la législation à l'époque de Nixon, étaient une pure mesure de compression des coûts, sans égard à la qualité médicale [10]. Mais aujourd'hui l'effet modérateur des coûts s'est envolé, la moindre raison n'étant pas que des décisions de refus de certaines prestations médicales ont été attaquées en justice avec succès par des patients. Cette tendance s'est encore renforcée avec l'énoncé des «droits du patient» («patient's bill of rights»).

Une étude en provenance de Harvard a montré que la fonction de "gardien du temple» des HMOs et des processus de Managed-Care du Plan Sanitaire de la Communauté de Harvard, et cela après 25 ans de fonctionnement, n'avait conduit à aucun changement substantiel dans la proportion des recours à son généraliste, respectivement à un spécialiste $[15,16]$. Pour cette raison, on cherche actuellement aux Etats-Unis de nouvelles voies, à savoir:

- participation du patient aux frais;

- réduction des frais administratifs;
- amélioration de la prise en charge du patient. On estime que près de la moitié des prestations représentent du «travail à double», donc du «surplace», comme par exemple la répétition des démarches diagnostiques.

\section{Pourquoi mettre sur pied un Réseau Suisse de I'Insuffisance Cardiaque?}

Heureusement, l'organisation sanitaire suisse ne souffre pas de certains défauts flagrants, qualitatifs et organisationnels, du système de santé américain. Pourtant, nous courons le risque d'imiter les erreurs du système américain ou britannique.

Pour ce qui est de l'insuffisance cardiaque, cependant, il existe encore, comme aussi dans d'autres pays, par exemple les Pays-Bas [17], des points faibles évidents, qui doivent être améliorés $[8,18]$. Tant le diagnostic que la thérapie s'écartent encore dans une proportion importante des directives basées sur les preuves et sont susceptibles d'amélioration. De plus, la place occupée par l'insuffisance cardiaque est sousestimée - bien que comparable avec les tumeurs malignes en termes de morbidité et de mortalité $[19,20]-$, en arguant qu'«il ne s'agit «que〉 d'une affection cardio-vasculaire et qu'elle survient fréquemment à un âge avancé, lorsqu'on se demande de toute façon jusqu'où aller avec les mesures diagnostiques et thérapeutiques. Naguère on traitait l'insuffisance cardiaque avec des médicaments bon marché, tels que digoxine, diurétiques et morphine, et cela marchait tout aussi bien». Voilà une opinion que l'auteur du présent article a souvent entendue.

Si l'on admet que notre devoir de médecins consiste toujours à soulager et si possible à prévenir la souffrance et que la politique suisse juge que la qualité de vie des insuffisants cardiaques mérite aussi d'être favorisée, alors il est logique de mettre en place un «réseau vertical et horizontal», où les partenaires de santé publique et le patient (y compris son entourage) travaillent ensemble et améliorent ainsi la qualité du traitement de l'insuffisance cardiaque.

Il est frappant de constater que des mesures diagnostiques et thérapeutiques efficaces et facilement accessibles sont appliquées de façon insuffisante. Comme exemples de mesures diagnostiques, mentionnons:

- recueillir une anamnèse ciblée des symptômes d'insuffisance cardiaque;

- réaliser une échocardiographie (Fr. 350.- à 400.-);

- déterminer le BNP ou le NT-pro-BNP (Fr. 60.à 70.-). 
Quant à la thérapeutique, on attache souvent trop peu d'importance aux mesures adjuvantes, comme p.ex. à l'information du patient, à des instructions sur la discipline thérapeutique, au maintien de l'euvolémie sous la propre responsabilité du patient (contrôle de poids, utilisation de diurétiques à la demande). L'indication péremptoire d'un traitement médicamenteux au long cours en présence d'une insuffisance cardiaque, conduit indubitablement à des frais considérables. T. Szucs a rédigé à ce sujet un article de revue très complet (cf. aussi la littérature déjà publiée [20, 21]).

\section{Qu'en est-il à l'étranger?}

\section{Allemagne}

La mise en réseau de l'information: c'est là la tâche principale des réseaux de compétence récemment créés pour les affections cardio-vasculaires. Les réseaux de compétence ont été créés pour la prise en charge de patients souffrant d'insuffisance cardiaque, de fibrillation auriculaire et de malformations cardiaques congénitales [22]. Comme exemple digne d'intérêt, citons une discipline non cardio-vasculaire qui utilise un réseau de compétence en Allemagne: la psychiatrie. Le «pacte de Nuremberg» contre la dépression a, par une «mise en réseau», contribué à une amélioration du diagnostic et du traitement de la dépression. Peu de temps après on constatait dans la région de Nuremberg un taux inférieur de suicides par rapport aux années précédant la «mise en réseau».

Le réseau de compétence allemand «Insuffisance cardiaque» s'est fixé les objectifs suivants:

- identifier les lacunes dans le diagnostic et la thérapeutique;

- encourager l'application des directives dans la pratique;

- surmonter les résistances psychologiques.

Un exemple simple de résistance psychologique est le refus du bêta-bloquant en début de traitement en raison de son association occasionnelle avec une diminution transitoire de la qualité de vie. Un exemple inverse est l'utilisation de substances inotropes positives, en raison d'une amélioration de l'état du patient à court terme, sans que le pronostic à long terme en soit amélioré [23]. On peut trouver plus de détails sur le réseau de compétence allemand pour l'insuffisance cardiaque sur le site www.kompetenznetz-herzinsuffizienz.de/start.

\section{Italie}

L'Italie s'est également mise en réseau pour améliorer la prise en charge de l'insuffisance cardiaque [24]. Par exemple, l'étude TEMISTOCLE (hearT failure EpideMIological STudy fadOi anmCo in itaLian pEople, FADOI = Federazione delle Associazione di Dirigenti Opsedalieri Internisti, ANMCO = Associazione Nazionale Medici Cardiologi Ospedalieri) a pour objectif d'évaluer la prise en charge actuelle de l'insuffisance cardiaque par les médecins et d'en éliminer les manquements par rapport aux directives. Il ne s'agit là aucunement de dénoncer des collègues, mais bien d'améliorer la qualité de la prise en charge. Divers projets (comprenant aussi les soins infirmiers lors d'insuffisance cardiaque) ont été élaborés [25-32]. Le site web www. heartfailure.it sera prochainement à même de fournir des rapports plus précis à ce sujet.

\section{Suède}

La Suède a accompli un travail exemplaire en faveur de l'insuffisance cardiaque. C'est ainsi que dès les années 70, elle a proposé l'emploi de bêtabloquants, et ce malgré la résistance du reste du monde [33]. Mais c'est surtout dans le domaine de l'amélioration de l'observance thérapeutique par l'utilisation de personnel soignant spécialisé en insuffisance cardiaque que la Suède a repris aujourd'hui un rôle d'avant-garde [34, 35]. Ce sont des infirmiers qui, dans un travail de pionner, ont mis sur pied le «Swedish Heart Failure Network» (www.v-i-c.nu/work_groups/ hjartsv.htm). A vrai dire il y a aussi des voix critiques, qui se plaignent d'un emploi abusif du réseau à des fins de politique professionnelle, un danger qui guette toute forme d'interaction interdisciplinaire [36]. Pourtant personne ne pourra formuler d'objection à l'amélioration de la formation et des compétences du personnel soignant. La question décisive, en Suède comme d'ailleurs chez nous en Suisse, est de savoir si on est préparé à une collaboration empreinte de respect mutuel.

\section{USA}

La Heart Failure Society of America (www. hfsa.org) a introduit des «National Heart Failure Awareness Weeks» dans le sens de campagnes de sensibilisation. Malheureusement les programmes sur l'insuffisance cardiaque aux Etats-Unis sont souvent des structures à but lucratif et qui dépendent de l'industrie (p. ex. www.HeartFailureReportCard.org). D'autres bonnes idées américaines quant à leur contenu peuvent être reformulées en fonction des intérêts de la Suisse. 


\section{Que fait la Suisse?}

En Suisse, il existe des programmes pour l'insuffisance cardiaque depuis les années 80 . Avec l'introduction de la transplantation cardiaque, on a institué des «consultations ambulatoires d'insuffisance cardiaque et de transplantation». A cette époque déjà, les patients étaient pris en charge selon les critères les plus modernes d'alors (y compris enseignement de l'observance thérapeutique et réhabilitation). Toutefois, la constitution d'un cercle d'experts qui traitent des aspects complexes de tous les patients insuffisants cardiaques n'a été vraiment réalisée qu'avec la fondation du groupe de travail «Insuffisance cardiaque» de la Société suisse de cardiologie en 1988.

En ce qui concerne la prise en charge médicale, il y a lieu d'intensifier la collaboration entre médecins de premier recours et spécialistes (cardiologues) d'une part, et d'autre part entre médecins praticiens et hospitaliers. Cela n'a certes pas été facilité par l'«émancipation» de la cardiologie, qui est devenue une spécialité à part entière. Les intérêts des patients doivent pourtant passer avant les considérations de politique professionnelle. Il en va de même, comme déjà mentionné, pour l'intégration du personnel soignant.

La mise en fonction de «systèmes d'autogestion de sa maladie» («Disease Management Programs» (à l'intérieur d'institutions de type

Figure 1

Partenaires du réseau suisse de l'insuffisance cardiaque. Le réseau comprend un «micro»-réseau, défini en premier lieu par les relations directes du patient. Le «macro»-réseau devrait se déployer à un niveau «stratégique» et être considéré comme une structure de prestations de service. En outre, le macroréseau devrait contribuer de façon constructive à améliorer la qualité de la prise en charge de l'insuffisance cardiaque en Suisse.

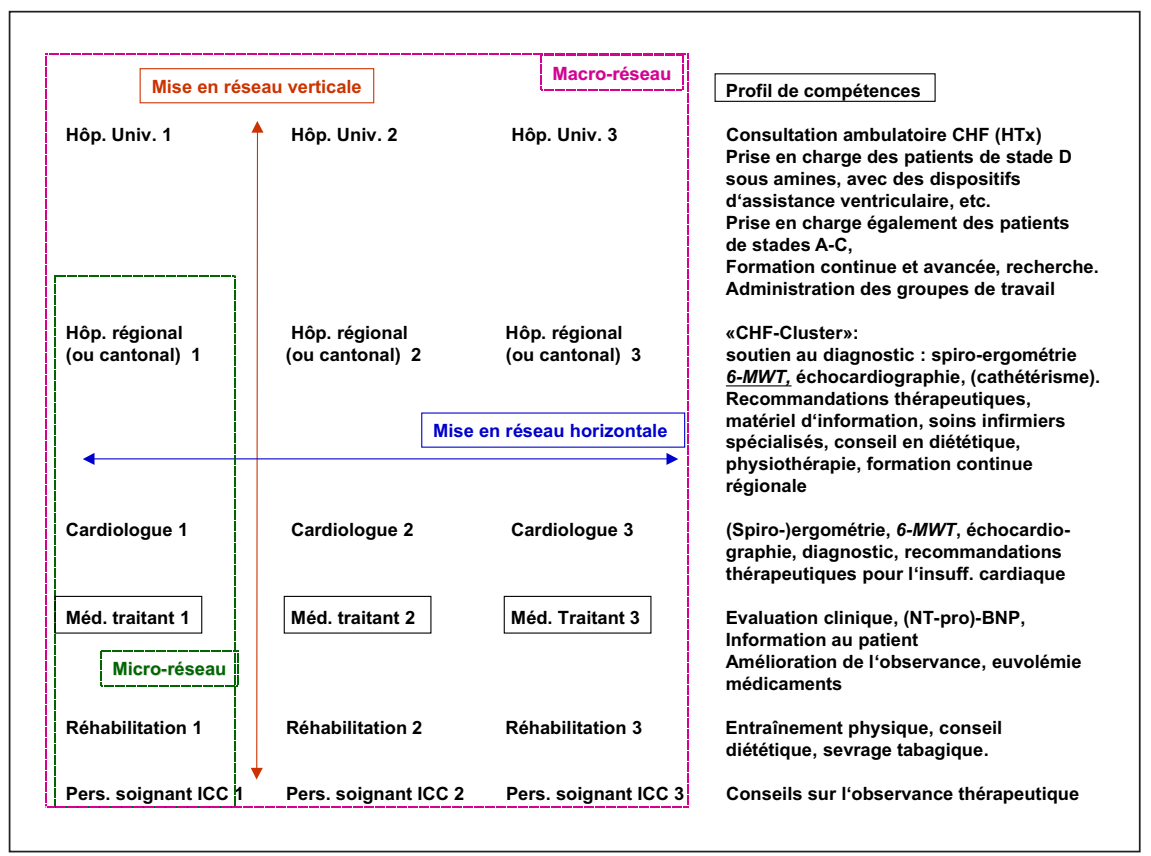

HMO) est contestée [37]. Le site médical MediX (www.medix.ch) a mis sur pied un programme de gestion de l'insuffisance cardiaque en association avec l'assurance Helsana [38]. Un autre exemple est celui de Medvantis (www.medi-24. ch). Le concept Medix, comme celui de Medvantis doivent être salués comme projets-pilotes. Mais ils ne correspondent qu'en partie aux souhaits du groupe de travail «Insuffisance cardiaque». Dans ce contexte, on oublie souvent qu'il existe déjà depuis plusieurs années des concepts thérapeutiques analogues dans les cliniques universitaires [39], sans grande publicité. Comme mentionné au début, l'effet d'un tel «Disease Management Program» sur la compression des coûts n'a pas encore pu être documenté, mais le temps d'observation est encore relativement limité. D'autre part, nos structures existantes - avec le médecin traitant comme plaque tournante, même en-dehors d'une HMO - conviennent parfaitement à la mise sur pied d'un réseau sur une base multidisciplinaire. C'est pourquoi nous allons présenter brièvement ci-dessous les composantes verticale et horizontale d'un réseau.

\section{Réseau vertical: collaboration entre hôpitaux et médecins établis}

Un réseau vertical - cela signifie favoriser le flux d'information entre hôpitaux et médecins traitants, étant admis que le médecin traitant est la plaque tournante [40] (fig. 1). Le système du médecin de famille est profondément implanté en Suisse, où il a atteint un haut niveau de qualité. Un des rôles du médecin de famille, encouragé par les caisses maladies, est celui de «gardien du temple», ce qui est censé réduire les coûts à un niveau raisonnable. Pour chaque région, il existe un nombre suffisant de cardiologues (en pratique privée ou en hôpital), pour encadrer le médecin traitant. Il en résulte un petit réseau de compétences régional («micro»-réseau). Grâce aux contacts personnels entre partenaires, les coûts administratifs de cette composante verticale peuvent être réduits au strict minimum.

L'équilibre subtil entre la médecine de premier recours et les hôpitaux régionaux et cantonaux, respectivement les hôpitaux universitaires à l'arrière-plan (fig. 2) ne doit pas être compromis par une défense excessive de intérêts propres des parties concernées (patients, caisses-maladies, personnel soignant, corps médical, administration, politiciens). L'idée de constituer un réseau ne doit pas «dévaloriser» les structures existantes, mais établir une base solide par complémentarité. 


\section{Réseau horizontal: multidisciplinarité, compétences des différentes catégories professionnelles et Assurance de Qualité des prestations}

Devraient être soumis à une mise en réseau horizontale les diverses cliniques universitaires, hôpitaux cantonaux et régionaux, centres de réhabilitation en clinique et ambulatoires, les médecins de famille et les spécialistes (cardiologues). Il est logique d'intégrer aussi les soins infirmiers dans le réseau horizontal (fig. 1). Le profil de compétence décrit dans la figure 1 n'est pas exhaustif. En fonction de la formation et des centres d'intérêt, certaines mesures diagnostiques et thérapeutiques peuvent être décalées verticalement.

La prise en charge interdisciplinaire de patients insuffisants cardiaques en intégrant le personnel infirmier est une idée qui a été initialement proposée par des cardiologues. En Suisse, elle s'est concrétisée lorsque nous avons été en contact avec le modèle suédois. Le modèle suédois a de nombreux avantages, mais il n'est pas incontesté, comme déjà mentionné [36]. En outre, la validité d'une prise en charge basée sur le téléphone doit être soigneusement vérifiée. Il n'est pas totalement justifiable, à nos yeux, de réduire le contact personnel avec le patient à des outils électroniques (portable avec des fonctions

Figure 2

Mise en réseau (microréseau). Le patient se meut dans la partie verticale du réseau. Le médecin traitant lui sert de plaque tournante. En fonction de son état, le patient est pris en charge par des spécialistes, respectivement par des établissements hospitaliers. Le patient n'est pas très concerné par la partie horizontale du réseau. Le réseau horizontal permet un franc échange d'opinions (discussion de cas [y c. avec participation des spécialistes de la partie verticale], échange d'opinions concernant la qualité de la prise en charge des patients, remplacements, formation continue et avancée). Le réseau vertical ne correspond pas à une structure hiérarchique. Il couvre plutôt des domaines de compétence différents (cf. fig. 1, profil de compétence).

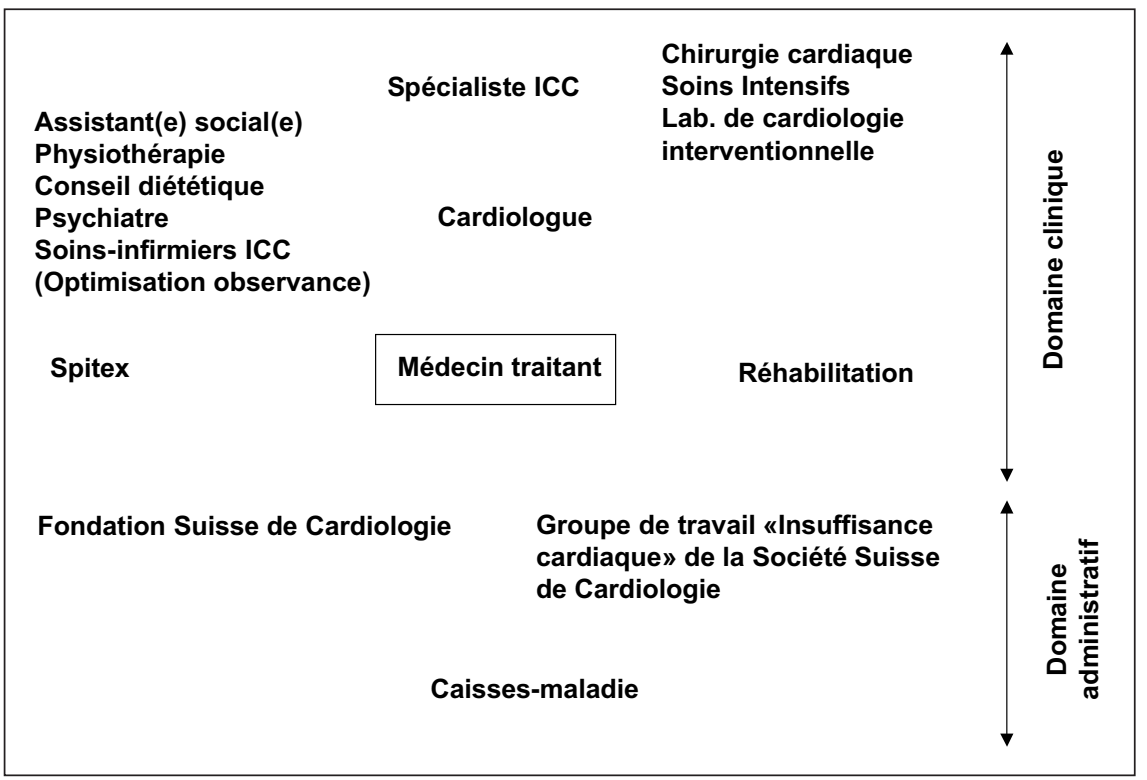

cardiaques, contrôle pondéral via PC et téléphone, respectivement «télénursing» [41]). Etant donné que le modèle suédois diffère du système suisse par sa structure, il ne peut pas être transposé sans subir des adaptations considérables. Il en va de même pour les Etats-Unis, qui s'offrent, et de loin, le système national de santé le plus onéreux, sans être à la pointe en ce qui concerne les soins de base.

Par interdisciplinarité, nous entendons le diagnostic, l'information initiale du patient et l'instauration du traitement par le médecin de façon correcte, des compléments d'information et la prise en charge par le personnel infirmier (observance thérapeutique) [42], le conseil diététique (apports en sel), la réhabilitation ambulatoire ou en clinique et, au besoin, le soutien psychologique. Beaucoup d'insuffisants cardiaques souffrent de dépression.

Le groupe de travail «Insuffisance cardiaque» et la Fondation Suisse de Cardiologie se sont fixé pour objectif de mettre sur pied des structures pour le perfectionnement et la formation continue du personnel spécialisé, ainsi que de la population et des patients (tab. 1).

C'est grâce à cette répartition des tâches et des compétences qu'on pourra former des structures bien définies, dans le sens d'un réseau. Une structure nouvelle, d'une grande importance à notre avis, est la possibilité d'avoir recours à un personnel infirmier ayant des connaissances spécialisées en insuffisance cardiaque [43-47]. Par analogie aux infirmières spécialisées en diabète ou en stomies, il est logique de dispenser à un personnel soignant motivé une formation spécifique dans la prise en charge de patients insuffisants cardiaques.

\section{Tableau 1}

Prestations de service du groupe de travail «Insuffisance cardiaque» de la Société Suisse de Cardiologie.

- site web www.heartfailure.ch (y compris les manifestations de formation continue);

- recommandations pour le diagnostic et le traitement de l'insuffisance cardiaque chronique [49];

- cours de formation «Insuffisance cardiaque» pour personnel soignant. Adresse de contact: Centre de Formation Hôpital de I'lle (AZI), Mme E. Vogt, Bereich Weiterbildungen, Murtenstrasse 10, 3010 Berne, tél. 03163243 27;

- distribution de matériel d'information pour patients: - kit pour patient de la Fondation de cardiologie (dès 2004),

CD-ROM;

- registre de l'insuffisance cardiaque;

- programme d'information sur l'insuffisance cardiaque auprès de la Fondation Suisse de Cardiologie (www.swissheart.ch). 
Les premiers infirmiers spécialisés en insuffisance cardiaque de Suisse ont été formés l'an dernier dans un centre de formation en soins infirmiers et ont été reconnus par un certificat délivré par ce centre, en collaboration avec le groupe de travail «Insuffisance cardiaque» de la Société Suisse de Cardiologie. Il ne s'agit pas là de mettre sur pied de nouvelles structures indépendantes (avec le risque de «perdre» le patient), mais bien de favoriser les composantes complémentaires de la prise en charge, qui compléteront la prise en charge centrale par le médecin de famille. Il doit paraître prochainement un synopsis sur cette possibilité de formation complémentaire pour le personnel soignant, intitulé «module insuffisance cardiaque», qui présentera le contenu de l'enseignement et les compétences professionnelles supplémentaires qui en résulteront [48]. En outre, le groupe de travail «Insuffisance cardiaque» édite des recommandations pour l'établissement de réseaux, textes qui doivent contribuer à établir des normes d'assurance de qualité [49]. Il y a donc lieu de mettre aussi sur pied les éléments locaux du réseau, spécifiques aux circonstances et règles du jeu locales. C'est à cela que nous aimerions vous encourager.

\section{Références}

1 Annas GJ. Women and children first. N Engl J Med 1995;333:1647-51.

2 Mohacsi P, Moschovitis G, Tanner H, Hess OM, Hullin R. Prevalence, increase and costs of heart failure. Heart Metabol 2001;14:9-16.

3 Stewart S, MacIntyre K, Capewell S, McMurray JJ. Heart Failure and the aging population: an increasing burden in the $21^{\text {st }}$ century? Heart 2003 ; 89:49-53.

4 Wanders A-C. Bundesamt für Statistik, Lausanne: Szenarien zur Bevölkerungsentwicklung der Schweiz 2000-2060. Markante Alterung zwischen 2005 und 2035. Pressemitteilung des Bundesamtes für Statistik 2001;0350-0103-20:1-10.

5 Szucs TD. Aging in Switzerland - epidemiologic and economic aspects. Kardiovask Med 2003; $6: 5-10$.

6 Burckhardt J. Das Gesundheitswesen in der Schweiz. Leistungen, Kosten, Preise. Pharma Information 2001:14-16.

7 McMurray J, McDonagh T, Morrison CE, Dargie HJ. Trends in hospitalization for heart failure in Scotland 1980-1990. Eur Heart J 1993;14:1158-62.

8 Moschovitis G, Zbinden S, Noll G, Hess OM, Mohacsi P. The Swiss Heart Failure Registry: a longitudinal follow-up survey. Kardiovask Med 2002;5(suppl 4):15 S.

9 Hunt S, Baker DW, Chin MH, et al. ACC/AHA Guidelines for the evaluation and management of chronic heart failure in the adult: executive summary. Circulation 2001;104:2996-3007.
10 Gasser DF. Managed Care: In den USA gescheitert. Medical Tribune 2002;35:20.

11 Discher CL, Klein D, Pierce L, Levine AB, Levine TB. Heart failure disease management: impact on hospital care, length of stay, and reimbursement. Congest Heart Fail 2003;9:77-83.

12 COPD Advisory Board. Disease Management COPD (erich.russi@dim.usz.ch). Koordinationsstelle MedLink, Postfach 108, CH-8808 Pfäffikon. www.diseasemanagement.ch 2002.

13 Diamond F. When HMOs can't say «No», physicians may have to. Manag Care 2001;10:26-31.

14 Lesser CS, Ginsburg PB, Devers KJ. The end of an era: what became of the «managed care revolution» in 2001. Health Serv Res 2003;38:337-55.

15 Ferris TG, Chang Y, Blumenthal D, Pearson SD. Leaving gatekeeping behind - effects of opening access to specialists for adults in a health maintenance organization. N Engl J Med 2001; 345:1312-17.

16 Lawrence D. Gatekeeping reconsidered. N Engl J Med 2001;345:1342-43.

17 Pont LG, van Gilst WH, Lok DJA, Kragten HJA, Haaijer-Ruskamp FM. Failure on behalf of the Dutch Working Group on Heart Failure. The relevance of heart failure severity for treatment with evidence-based pharmacotherapy in general practice. Eur J Heart Failure 2003;5:187-93.

18 Wagdi P, Vuilliomenet A, Kaufmann U, Richter M, Bertel O. Ungenügende Behandlungsdisziplin, Patienteninformation und Medikamentenverschreibung als Ursachen für die Notfallhospitalisation bei chronisch herzinsuffizienten Patienten. Schweiz Med Wochenschr 1993;123:108-12.

19 Stewart S, MacIntyre K, Hole DJ, Capewell S, McMurray J. More «malignant» than cancer? Fiveyear survival following a first admission for heart failure. Eur J Heart Failure 2001;3:315-22.

20 Szucs TD, Belisari A, Mantovani LG. Can Europe afford not to implement evidence-based medicine in heart failure. Eur Heart J 1998; 19(Suppl L): L22-L27.

21 Szucs TD, Goedde M, Berger K, Kiowski W. Wirtschaftlichkeit der ACE-Hemmung in der Therapie der chronischen Herzinsuffizienz in der Schweiz: Evaluation auf der Grundlage der SOLVD-Studie. Schweiz Med Wochenschr 1997;127:1234-41.

22 Dietz R, Klawki R. Kompetenznetze - was haben wir davon? Cardio News (Deutsche Gesellschaft für Kardiologie) 2003;6:18.

23 Packer M, Cerver JR, Rodeheffer RJ, Ivanhoe RJ, DiBianco R, Zeldis SM for the PROMISE Study Research Group. Effect of oral milrinone on mortality in severe chronic heart failure. N Engl J Med 1991;325:1468-75.

24 Porcu M, Opasisch C, Scherillo M, et al. Improving practice patterns in heart failure through a national cardiological network: the case of ACEinhibitors. Ital Heart J 2002;3:730-7.

25 Del Sindaco D, Pulignano G, Maggioni AP, et al Assessment of disability and quality of life in elderly patients with heart failure: data from the Italian Network on Chronic Heart Failure (INCHF) - Nursing Study. Eur J Heart Failure 2000; 2:100. 
26 Del Sindaco D, Pulignano G, Maggioni AP, et al Functional, cognitive and socio-environmental determinants of hospital admission in elderly outpatients with heart failure. Eur J Heart Failure 2000;2:101.

27 Pulignano G, Del Sindaco D, Maggioni AP, et al. In the elderly the impact on disability and quality of life is similar in heart failure with preserved versus impaired systolic ventricular function. Eur J Heart Failure 2000;2:100.

28 Pulignano G, Del Sindaco D, Maggioni AP, et al. Predictors of 1 year mortality and mode of death in 1033 elderly outpatients with heart failure: Data from Italian Network on congestive heart failure. Eur J Heart Failure 2000;2:102.

29 Faggiano P, Domenichini D, Giusti A, et al. Prescription patterns of diuretics in chronic heart failure: Data from the Italian Network on Congestive Heart Failure (IN-CHF) database. Eur J Heart Failure 2000;2:22

30 Pulignano G, Del Sindaco D, Maggioni AP, et al. The Minnesota living with heart failure questionnaire correlates with disability and clinical parameters in elderly patients with heart failure. Eur J Heart Failure 2000;2:101-2.

31 Faggiano P, Cacciatore G, Gorini M, et al. Use of aldosterone receptor blockers in chronic heart failure before the results of RALES Study. Data from Italian Network on Congestive Heart Failure (IN-CHF). Eur J Heart Failure 2000;2:22.

32 Maggioni AP, Masson S, Lucci D, et al. Clinical correlates of elevated plasma natriuretic peptides and Big endothelin-1 in a population of ambulatory patients. Eur J Heart Failure 2000;2:48.

33 Waagstein F, Hjalmarson A, Varnauskas E, Wallentin I. Effect of chronic beta-adrenergic receptor blockade in congestive cardiomyopathy. Br Heart J 1975;37:1022-36.

34 Herzog A, Lind L. Network solutions for home health care applications. Technol Health Care 2003;11:77-87.

35 Strömberg A, Mårtensson J, Fridlund B, Dahlström U. Nurse-led heart failure clinics in Sweden. Eur J Heart Failure 2001;3:139-44.

36 Cline C. Nurse-led clinics for heart failure in Sweden - doing the right thing? Eur J Heart Failure 2002;4:393-4.

37 de Haller J. Schweizerische Gesellschaft für Allgemeinmedizin, Helsana und Disease Management. PrimaryCare 2003;3:457-8.

38 Götschi AS, Weber A. Herzprogramm - Leben mit dem kranken Herz. Erfahrungen mit einem
Diseasemanagementprogramm für Patienten mit Herzinsuffizienz. Schweiz Ärztezeitung 2002; 38:1987-92.

39 Atar D, Gustafsson F, Galatius S, Hildebrandt PR. Das Konzept der Herzinsuffizienz-Klinik: Integriertes diagnostisches und therapeutisches Vorgehen bei Patienten mit linksventrikulärer systolischer Dysfunktion. Kardiovask Med 2002; 5:421-30.

40 Boos L, Dieckmann M. Spitäler als Nuklei von Gesundheitsnetzen. Neue Zürcher Zeitung 2003; $54: 27$.

41 Jerant AF, Azari R, Martinez C, Nesbitt TS. A randomized trial of telenursing to reduce hospitalization for heart failure: patient-centered outcomes and nursing indicators. Home Health Care Serv Q 2003;22:1-20

42 Strömberg A, Ahlén H, Fridlund B, Dahlström U. Interactive education on CD-ROM - a new tool in the education of heart failure patients. Patient Educ Couns 2001.

43 Smith M, Irving JB. Managing heart failure in the community; role of the nurse specialist. Health Bull 2001;59:340-2.

44 Strömberg A, Dahlström U, Fridlund B. Computer-based education for patients with chronic heart failure. A randomised, controlled multicentre study of the effects on knowledge, compliance and quality of life. Submitted (published in 47).

45 Strömberg A, Mårtensson J, Fridlund B, Levin L- $\AA$, Karlsson J-E, Dahlström U. Nurse-led heart failure clinics improve survival and self-care behaviour in patients with heart failure. Results from a prospective, randomised study. Submitted (published in 47).

46 Strömberg A, Broström A, Dahlström U, Fridlund B. Factors influencing patient compliance with therapeutic regimens in chronic heart failure: a critical incident technique analysis. Heart Lung 1999;28:334-41.

47 Strömberg A. Caring for patients with chronic heart failure - with focus on patient education and nurse-led heart failure clinics: Linköping Universitet, Sweden, 2001.

48 Mohacsi P, Vogt E. Herzinsuffizienz-Kurs für Pflegende. Kardiovask Med (in press).

49 Arbeitsgruppe für Herzinsuffizienz der Schweizerischen Gesellschaft für Kardiologie. Empfehlungen zur Diagnose und Behandlung der chronischen Herzinsuffizienz. Schweiz Ärztezeitung 2002; 83:1233-42.

\section{$11^{\text {e }}$ Réunion de Gstaad 2004 \\ de la Fondation Suisse de Cardiologie \\ 29 janvier au $1^{\text {er }}$ février 2004}

Pour plus d'information: www.congress-info.ch/gstaad2004/

Patronat du congrès: Fondation Suisse de Cardiologie et Swiss Society of Cardiology

Sponsor principal du congrès: AstraZeneca

Co-sponsor du congrès: 